The smaller and more inconspicuous animals did not escape Dampier's notice, for he tells of the two sorts of land crab, the white and the black; "black Crabs are commonly fat and full of Eggs; they are also accounted the better Meat, though both sorts are very good". He also knew Limulus, the primitive horse-shoe crab. Near Campeachy "in the Surf of the Sea, close by the shore, you find abundance of Shell-fish, called by the English, Horse Hoofs, because the under part or Belly of the Fish is flat, and somewhat resembling that Figure in shape and Magnitude; but the Back is round like a Turtles; the Shell is thin and brittle, like a Lobsters; with many small Claws: and by report they are very good Meat; but I have never tasted of them myself. . . . Here are also a sort of Spiders of a prodigious size ... they have two Teeth, or rather Horns an Inch and a half, or two Inches long ... black as Jet, smooth as Glass, and their small end sharp as a Thorn. These Teeth we often preserve. Some wear them in their 'Tobacco pouches to prick their Pipes. Others preserve them for Tooth-Picliers".

In the account of his voyage to Australia or "New Holland" in the Roebuck in 1699, Dampier gives even fuller details of the natural history, the birds, beasts, and especially the fishes, and also of the plants that he found. He illustrates many of these in copper plates that, considering the times, are remarkably good. Many of the species are quite easily identified from his sketches, and it could well be claimed that he was one of the first traveller-naturalists.

As is only natural, Dampier gives the colours of the birds and other creatures that he saw, and his descriptions are correct; yet one may doubt whether his colour perception was complete. When he was at 'Tonquin he saw the galleys of the king's navy which were commanded by a general who "was a great Mandarin. ... There were two more great Officers under him, each in a Vessel by himself. These three had Flags of distinction: the first was yellow, the second blue, the third red or green". Can it be that Dampier was colour-blind, and could not distinguish red from green?

\section{BRITISH MYCOLOGICAL SOCIETY PLANT PATHOLOGY FIELD DAY}

$\mathrm{T}$ HE twenty-sixth annual plant pathology field day of the British Mycological Society was held on July 4 in the Mycology Section of the Hawthorndale Laboratories, Bracknell, Berkshire, of Imperial Chemical Industries, Ltd. Sixty-six members attended.

Mr. R. V. Tipler, senior mycologist, reviewing the functions of the laboratories, stated that the main task was the evaluation of chemicals produced in the research departments of I.C.I. manufacturing divisions for possible use in controlling crop diseases, pests and wastage caused by bacteria, fungi, insects and eelworms in agriculture, horticulture and industry. The Mycology Section was concerned with prevention or control of plant diseases and crop wastage and with industrial microbiological problems. The demonstrations were arranged to illustrate the scope of the Section's activities in the field of bioassay. They showed the sequence of testing from first-stage sorting by in vitro methods for general antifungal and antibacterial activity to second-stage sorting by in vivo methods in laboratory, glasshouse and field to discover applications for promising chemicals in industry and as seed, soil and foliage fungicides. Consolidation work, not capable of being shown at the Laboratories, followed under practical conditions at home and overseas with selected materials, under control of the research staff.

Miss M. E. Kennedy demonstrated methods of maintaining the culture collection. In order to reduce subculturing and minimize possible variations in reactions of test fungi to chemicals or pathogenicity to plants the mineral oil and freeze-drying methods of preservation were used. The former was more suitable for mycelial and non-sporing forms and consisted of culture on agar slopes covered to a depth of $1 \mathrm{~cm}$. above the medium with a sterilized mineral oil. In the latter, suspensions of fungal spores $\mathrm{Or}^{2}$ bacteria, with a protective colloid, were dried in vacuo from the frozen state. Standard reference cultures had been preserved unchanged in the characteristics under study for more than four years. The Hawthorndale method of inducing profuse sporulation of Alternaria solani was demonstrated. Controlled ultra-violet irradiation of mycelial cultures on agar reliably produced spores within eighteen hours. The method had been in regular use for three and a half years.

Mr. J. L. Charlton showed agar plate and spore germination techniques for in vitro screening of chemicals. The first method, based on serial dilution of chemicals in an agar medium which was then inoculated with a range of fungi or bacteria, rapidly placed new chemicals in activity categories. Active chemicals were investigated more precisely by the second method, using spores of Alternaria solani, Venturia incequalis or Botrytis allii. This method was particularly suitable for investigating groups of related chemicals and effect of formulation on antifungal activity. An auxanographic method of demonstrating interactions between effects of chemicals on fungi was on view. Morphological effects of chemicals on germinating spores of Botrytis allii were also shown.

Mr. J. N. Turner had on view the miniature kiln test permitting quantitative comparison of chemicals for control of timber sap-stain and moulding, as used for second-stage evaluation of potential industrial fungicides. Notable features were the use of paired surfaces for treatment versus control comparisons and the high degree of replication achieved within a small space. Tests were seen in progress comparing the British Standards and the Pechmann--Schaile methods for laboratory evaluation of timber preservatives. Mycelial mat and soil burial methods for assessing textile preservatives were shown, together with investigations on the effect of leaching and weathering on degree and duration of proofing. Species of fungi responsible for moulding and staining of baled rubber were exhibited.

Miss S. E. Parkinson illustrated with specimens and cultures of the microflora isolated from them the extent and types of damage to leathercloths caused by micro-organisms.

Types of slimes encountered in paper mills were shown, with cultures of bacteria and fungi responsible for their production. Efficiency of control by organomercurial compounds depended, inter alia, upon the stock, as its composition affected both the type of slime and the absorption and inactivation of mercury by the pulp. Techniques demonstrating bioassay of active mercury in pulp and white-water included two plate methods using Cladosporium herbarum and a turbidimetric method using Lactobacillus helveticus. 
A sensitivity allowing $0.05 \mu \mathrm{gm}$. per gm. of active mercury to be detected was achieved.

Methods of determining the resistance of slime organisms to chemicals were also shown.

Mr. I. W. McC. Callan dealt with tests for evaluating chemicals for use as seed fungicides. Laboratory phytotoxicity tests on wheat supplemented glasshouse disease control trials using oats leaf spot (Pyrenophora aveno) and pea foot rot (Pythium ultimum) for determination of disinfectant and protectant properties respectively. Examples of these standardized replicated experiments were seen. In the experiment garden were demonstrations of the application of seed treatments for controlling seedand soil-borne diseases, particularly seedling rots, of peas, maize, flax, sugar beet, spinach and cucurbits. 'The extensive 'rod-row' trials which wore seen illustrated a replicated block technique based on single rows used for field assay of chemicals for controlling cereal diseases such as wheat bunt and oats and barley smuts.

Machinery for commercial application of dry and wet seed dressings was demonstrated, and in the laboratory an exhibit of a modification of Machacek's agar-sheet technique showed strikingly the extent of variation of load of toxicant on individual seeds obtainable by varying the seed-dressing formulation and the type of machinery used.

Threshing and winnowing equipment developed for accurate estimation of yields from metre-square cereal sampling plots was also shown.

Dr. J. Stubbs had exhibits of a range of plant diseases which were kept in culture and used for glasshouse evaluation of foliage fungicides. These included Alternaria solani and Phytophthora infestans on tomato, Uromyces faboe, Botrytis cinerea and $B$. fabce on broad bean, Puccinia antirrhini on antirrhinum, Erysiphe graminis on oat, E. cichoracearum on marrow, and Septoria apii on celery. The methods of using $A$. solani on tomato for detection and measurement of systemic activity were shown, using griseofulvin as example of an active systemic fungicide. They involved absorption by the plant of chemicals applied to roots or leaves. A 0.05 per cent solution of griseofulvin applied to the soil in which small tomato plants were growing afforded complete protection against disease, whereas the same concentration applied to foliage gave only partial disease control on leaves which had been shielded from the spray.

Mr. J. A. W. Turner dealt in detail with the equipment and methods used in the routine $A$. solani test as developed for the first in vivo assessment of potential foliage fungicides and for comparison of effects of formulation (for example, of copper and thiram fungicides) on disease control.

Three tomato plants were sprayed with each of a range of concentrations of a chemical under conditions giving standard deposits. After twenty-four hours drying, plants were inoculated by spraying with $A$. solani spore suspension, incubated at 100 per cent relative humidity for a further twenty-four hours and transferred to the glasshouse. Disease counts were made after three days. ED 50 and ED 95 values were obtained graphically from data from three such tests made at approximately weekly intervals. Thiram was used as standard reference fungicide.

Chemicals active against $A$. solani were then examined further, as required, on the range of diseases demonstrated by Dr. Stubbs, before field evaluation commenced.
Mr. C. E. D. Smith described investigations on the control of Fusarium patch of turf, storage diseases of gladiolus corms and narcissus basal rot (Fusarium bulbigenum).

Techniques for the bioassay of soil fungicides were shown, based on selective isolation of Pythium ultimum and Thielaviopsis basicola from soils containing the viable pathogens. These were coupled with further experiments to determine persistence of the chemicals in soil and their phytotoxicity. Successful chemicals were tested against clubroot (Plasmodiophora brassica) by a rapid laboratory method, and against Colletotrichum atramentarium attacking tomatoes. Demonstrations of this last disease included a character for its early diagnosis and a selective method for isolation of the pathogen.

A tour of the eight-acre experiment garden and trials field enabled further aspects of work described in the laboratories to be seen. These included gladiolus corm and narcissus bulb experiments, potato and tomato blight spraying plots and an orchard for investigation of apple, pear and bush fruit diseases.

R. V. TIPLER

\section{MELLON INSTITUTE ANNUAL REPORT FOR $1951-52$}

$7 \mathrm{HE}$ annual report of the President of the Mellon Institute to the Board of Irustees for the year ended February 29, 1952*, which is published in the Research Proceedings of the Mellon Institute, includes a full list of fellowships in operation during March 1,1951-March 1, 1952. Of the Institute's expenditure of $3,835,314$ dollars during the year, 727,654 dollars went to the support of investigations in pure science in the Institute's research departments and on nine fellowships, and these projects occupied 117 members of the Institute's staff. In applied science, seventyseven other fellowships occupied 441 members, and the servicing staff of the Institute numbered 162 . New fellowships put into operation during the year are concerned, respectively, with problems of air purification; the development of new chemicals and speciality products, other than fuels and lubricants, used in the operation and maintenance of automobile and aeronautical vehicles and equipment; the technology of glazed and unglazed structural clay products; the combustion of gaseous fuels; the fundamental molecular properties which limit the breaking strength of glass; and the thermostability of active dry yeast.

The Gas Purification Fellowship, which originated in 1939 and was terminated during the year, has led to the development of various new analytical methods, for example, for the rapid determination of hydrogen sulphide and mercaptan sulphur in gases and aqueous solutions, and to a process for reducing the organic sulphur content of coke-oven gas to about $0.5 \mathrm{gm} . / \mathrm{cu}$. ft. by scrubbing with an organic base. The Pipe Joint Fellowship, which also has been concluded, has led to the formulation of a product providing adequate thread lubrication during joint make-up and capable of forming a pressure-tight seal in the coupled joints. Other fellowships terminated during the year were the multiple fellowships dealing

* Research Proceedings of the Mellon Institute, 1951-1952 (Annual Report Series No. 39): Annual Report of the President, Edward $R$. Weidlein, to the Board of Trustees of the Institution for the Fiscal Year ended February 29, 1952. Pp. vi +50. (Pittsburgh, Pa.: Mellon Institute, 1952.) 